\title{
BMJ Open Direct transfer to angiosuite for patients with severe acute stroke treated with thrombectomy: the multicentre randomised controlled DIRECT ANGIO trial protocol
}

\author{
Nolwenn Riou-Comte, ${ }^{1}$ François Zhu, ${ }^{2,3}$ Aboubaker Cherifi, ${ }^{4}$ \\ Sébastien Richard (D) , ${ }^{1}$ Lionel Nace, ${ }^{5}$ Gérard Audibert, ${ }^{6}$ Hamza Achit, ${ }^{7}$ \\ Vincent Costalat, ${ }^{8}$ Caroline Arquizan, ${ }^{9}$ Olivier Beaufils, ${ }^{10}$ Arturo Consoli, ${ }^{11,12}$ \\ Bertrand Lapergue, ${ }^{13}$ Thomas Loeb, ${ }^{14}$ Aymeric Rouchaud, ${ }^{15}$ Francisco Macian, ${ }^{16}$ \\ Dominique Cailloce, ${ }^{17}$ Alessandra Biondi, ${ }^{18}$ Thierry Moulin, ${ }^{19}$ Thibaut Desmettre, ${ }^{20}$ \\ Gaultier Marnat, ${ }^{21}$ Igor Sibon, ${ }^{22}$ Xavier Combes, ${ }^{23}$ Ariel Pablo Lebedinsky, ${ }^{24}$ \\ Francis Vuillemet, ${ }^{25}$ Nicolas Kempf ${ }^{26}$ Laurent Pierot, ${ }^{27}$ Solene Moulin, ${ }^{28}$ \\ Philippe Lemmel, ${ }^{29}$ Mikael Mazighi, ${ }^{30}$ Raphael Blanc, ${ }^{30}$ Candice Sabben, ${ }^{31}$ \\ Eric Schluck, ${ }^{32}$ Serge Bracard, ${ }^{2,3}$ René Anxionnat, ${ }^{2,3}$ Francis Guillemin, ${ }^{33}$ \\ Gabriela Hossu, ${ }^{3,4}$ Benjamin Gory (D) ,2,3 DIRECT ANGIO Investigators
}

To cite: Riou-Comte N, Zhu F, Cherifi A, et al. Direct transfer to angiosuite for patients with severe acute stroke treated with thrombectomy: the multicentre randomised controlled DIRECT ANGI0 trial protocol. BMJ Open 2021;11:e040522. doi:10.1136/ bmjopen-2020-040522

- Prepublication history for this paper is available online. To view these files, please visit the journal online (http://dx.doi org/10.1136/bmjopen-2020040522).

Received 22 May 2020 Revised 19 0ctober 2020 Accepted 22 October 2020

Check for updates

(c) Author(s) (or their employer(s)) 2021. Re-use permitted under CC BY-NC. No commercial re-use. See rights and permissions. Published by BMJ.

For numbered affiliations see end of article.

Correspondence to Professor Benjamin Gory; benjagory@gmail.com

\section{ABSTRACT}

Introduction Mechanical thrombectomy (MT) increases functional independence in patients with acute ischaemic stroke with anterior circulation large vessel occlusion (LVO), and the probability to achieve functional independence decreases by $20 \%$ for each 1 -hour delay to reperfusion. Therefore, we aim to investigate whether direct angiosuite transfer (DAT) is superior to standard imaging/emergency department-based management in achieving 90-day functional independence in patients presenting with an acute severe neurological deficit likely due to LVO and requiring emergent treatment with MT.

Methods and analysis DIRECT ANGIO (Effect of DIRECT transfer to ANGIOsuite on functional outcome in patient with severe acute stroke treated with thrombectomy: the randomised DIRECT ANGIO Trial) trial is an investigatorinitiated, multicentre, prospective, randomised, open-label, blinded endpoint (PROBE) study. Eligibility requires a patient $\leq 75$ years, pre-stroke modified Rankin Scale (mRS) 0-2, presenting an acute severe neurological deficit and admitted within 5 hours of symptoms onset in an endovascular-capable centre. A total of 208 patients are randomly allocated in a 1:1 ratio to DAT or standard management. The primary outcome is the rate of patients achieving a functional independence, assessed as mRS 0-2 at 90 days. Secondary endpoints include patients presenting confirmed LV0, patients eligible to intravenous thrombolysis alone, patients with intracerebral haemorrhage and stroke-mimics, intrahospital time metrics, early neurological improvement (reduction in National Institutes of Health Stroke Scale by $\geq 8$ points or reaching $0-1$ at 24 hours) and $\mathrm{mRS}$ overall distribution at 90 days and 12 months. Safety outcomes are death and intracerebral haemorrhage transformation. Medico-economics analyses include healthrelated quality of life and cost utility assessment.

\section{Strengths and limitations of this study}

- DIRECT ANGIO trial is the first multicentre randomised clinical trial to directly compare direct angiosuite transfer (DAT) versus standard management for highly suspected patients with anterior circulation large vessel occlusion ischaemic stroke.

- DIRECT ANGIO aims to provide further evidence of the clinical benefit of DAT, as well as socioeconomic positive impact for the global health system.

- The multicentre setting and large pragmatic inclusions criteria compatible with current clinical practice and recommendations will allow external validity.

- Primary outcome measure will allow evaluation of functional independence at 90 days.

- Secondary outcomes will measure the safety and medico-economic impact of DAT.

Ethics and dissemination The DIRECT ANGIO trial was approved by the ethics committee of lle de France 1. Study began in April 2020. Results will be published in an international peer-reviewed medical journal.

Trial registration number NCT03969511.

\section{INTRODUCTION}

\section{Background and rationale}

Strokes remain a large cause of death and disability, and prevalence of large vessel occlusion (LVO) among patients with suspected acute ischaemic stroke ranged from $13 \%$ to $52 \%$, with overall prevalence of $30.0 \%$. ${ }^{1}$ Mechanical thrombectomy (MT) has become 
the standard of care for reperfusion therapies in acute anterior LVO strokes, ${ }^{2}$ and is strongly dependent on time with $20 \%$ decreased probability of functional independence for each 1-hour delay to reperfusion. ${ }^{3}$ The Highly Effective Reperfusion evaluated in Multiple Endovascular Stroke Trials (HERMES) meta-analysis demonstrated that prognosis is directly related to combined ischaemic core volume with age and expected imaging-to-reperfusion time after successful reperfusion. ${ }^{4}$ While the stroke network reorganisation reduced symptoms-to-needle time and increased accessibility to endovascular-capable centres, it is currently crucial to achieve fast triage and initiation of endovascular therapy. To date, patients with a suspected stroke are first admitted in the radiology/emergency department and secondary transfer to the angiosuite for MT if LVO is confirmed. This approach results in prolonged delays in delivering definitive therapy in the setting of LVO, whereas the angiosuite has imaging facilities to rule out intracranial haemorrhage (ICH) and confirm proximal arterial occlusion (cone beam CT (CBCT)), and therefore the ability to triage patients. Retrospective studies reported a clinical benefit of a direct angiosuite transfer (DAT) ${ }^{5-7}$ The aim of DIRECT ANGIO (Effect of DIRECT transfer to ANGIOsuite on functional outcome in patient with severe acute stroke treated with thrombectomy: the randomised DIRECT ANGIO Trial) trial is to compare the effectiveness and safety of DAT versus conventional management in patients presenting an acute severe neurological deficit and thus mainly due to LVO eligible to MT.

\section{Objectives}

Primary objective

The primary objective of the study is to determine whether DAT compared with conventional management is associated with improved 90-day functional independence in patients presenting with prehospital acute severe neurological deficit likely to require treatment with MT. Functional independence is defined as a modified Rankin Scale (mRS) score of 0-2 at 90 days.

\section{Secondary objectives}

The study will also explore the feasibility, efficacy and safety of DAT, as well as cost-utility assessment.

\section{Trial design}

The DIRECT ANGIO trial is a prospective, multicentre, randomised, open-label, blinded-endpoint (PROBE), two-arm, clinical trial to compare the effectiveness and safety of DAT versus standard management in patients with acute prehospital severe neurological deficit suspected to LVO of the anterior circulation.

\section{Consort diagram}

Figure 1 shows the shows the Consolidated Standards of Reporting Trials (CONSORT) diagram of the DIRECT ANGIO trial. ${ }^{8}$

\section{METHODS AND ANALYSIS}

\section{Participants, interventions and outcomes}

This manuscript was written in accordance with the Standard Protocol Items: Recommendations for Interventional Trials guidelines. ${ }^{9}$

\section{Study setting}

The DIRECT ANGIO trial takes place in nine comprehensive stroke centres in France (CHRU-Nancy, CHU Besançon, CH Colmar, CHU Strasbourg, CHU Reims, Foch, CHRU Montpellier, CHU Limoges and CHU Bordeaux).

\section{Eligibility criteria}

\section{Inclusion criteria}

Adult patients $\leq 75$ years old, pre-stroke mRS 0-2, with acute severe neurological deficit at prehospital stage and directly admitted at an endovascular-capable centre within 5 hours of symptoms onset and who meet all eligibility criteria are considered for study enrolment. Secondary transfer patients are not eligible in the trial. As the study objective is to target a completely autonomous population that can be assumed to have neither cognitive problems, nor a history of stroke age was limited to 75 years old. Table 1 lists the inclusion and exclusion criteria.

As of the phone call from the emergency rescue service, inclusion and exclusion criteria are checked and then the patient randomise during the hospital travel (before admission). We are modifying the timing of randomisation. Randomisation will be performed after severe stroke confirmation by a neurologist immediately after admission (version 3.0). Oral informed consent will be sought via telephone conversations from patient or from their relatives. Emergency consent procedure may be considered if consent is not possible by the subject or a proxy. Written informed consent for continuation will be then collected as soon as possible, within 3 months.

\section{Interventions}

Experimental arm (DAT approach)

On arrival in angiosuite and after rapid neurological examination (using National Institutes of Health Stroke Scale (NIHSS) and mRS scores) and blood sample, the patient undergoes CBCT in order to exclude non-ischaemic stroke and angiogram to confirm LVO (tandem, intracranial internal carotid artery, M1 or proximal M2 segment of the middle cerebral artery, basilar artery or P1 segment of the posterior cerebral artery). Several managements can be performed:

1. Whatever the NIHSS score at admission, patients with no ICH and with LVO were treated with MT and, if eligible, with intravenous recombinant tissue plasminogen activator (IV rt-PA) as soon as possible. A low Alberta Stroke Program Early CT Scale (ASPECTS) or low collateral score was not an exclusion criterion for MT.

2. Patients with no ICH and with a distal vessel occlusion were treated with IV rt-PA alone, if eligible. 


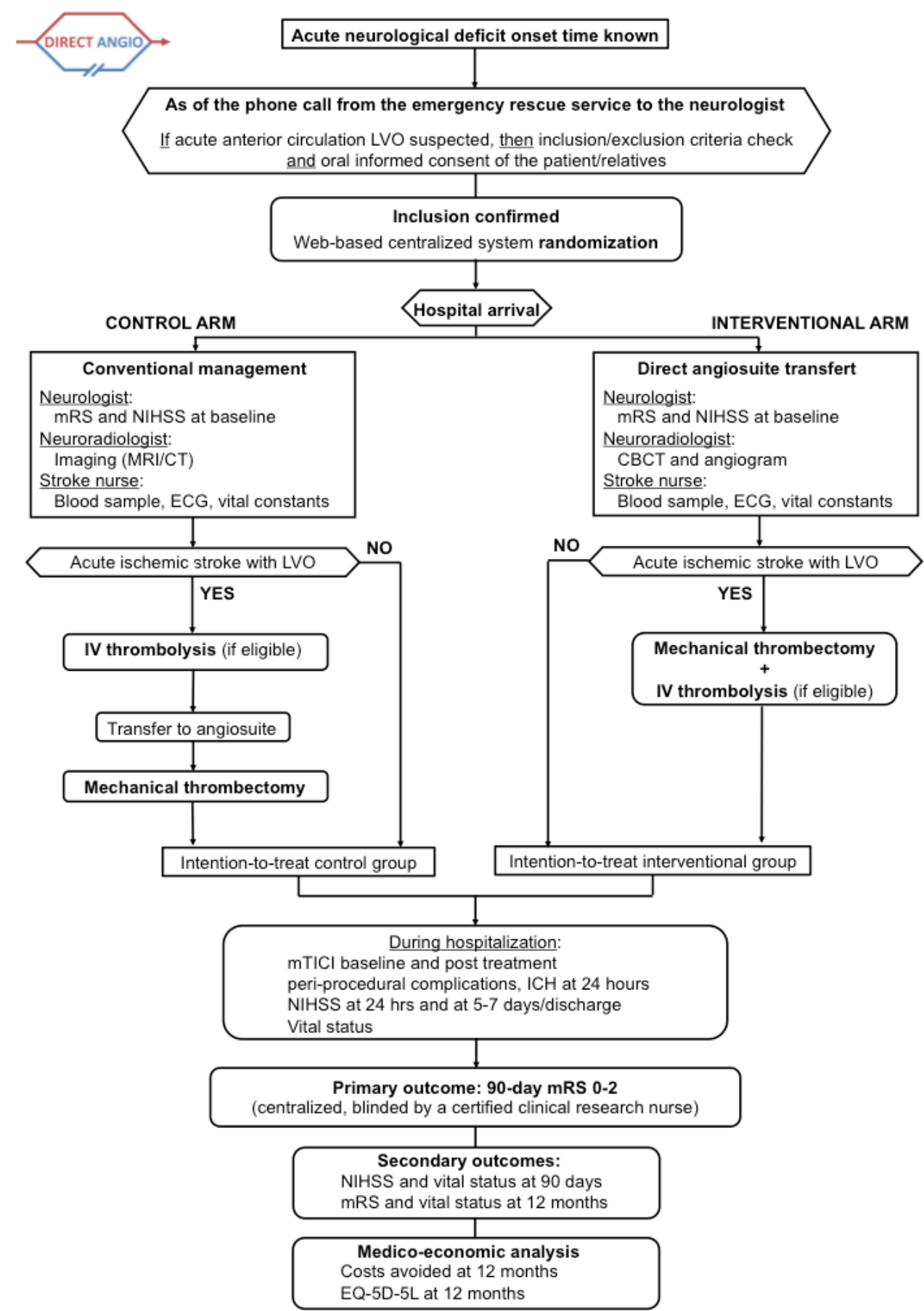

Figure 1 CONSORT (Consolidated Standards of Reporting Trials) diagram of the DIRECT ANGIO trial illustrating the randomisation and flow of patients in the study. CBCT, cone beam CT; EQ-5D-5L, 5 dimensions and 5 levels EuroQol questionnaire; ICH, intracerebral haemorrhage; IV, intravenous; LVO, large vessel occlusion; mRS, modified Rankin Scale; $\mathrm{mTICl}$, modified Thrombolysis In Cerebral Infarction; NIHSS, National Institutes of Health Stroke Scale.

3. Patients with no ICH and with no arterial occlusion were started on IV rt-PA, if eligible, and received an additional stroke imaging (MRI or CT) to decide on further treatment.

4. Patients with an ICH and no occlusion were treated as per institutional standards.

5. Patients with an ICH and LVO were treated with MT after an individualised case discussion between neurologist, neuroradiologist and patient or his/her proxy.
However, the subject will remain in the intention-totreat population.

Control arm (conventional approach)

Arrival is in the imaging/emergency department, and after neurological examination and blood sample, the patient undergoes stroke imaging (MRI or CT). After LVO confirmation, the patient is treated with IV rt-PA, if eligible, and transfer to angiosuite for MT as soon as 
Table 1 DIRECT ANGIO inclusion and exclusion criteria

\begin{tabular}{ll}
\hline Inclusion & Exclusion \\
\hline Adult $\leq 75$ years & Severe allergy to contrast agents \\
Prestroke mRS $0-2$ & Pregnant or breastfeeding women \\
Acute severe neurological deficit at hospital admission confirmed & Consent refusal or opposition of the relatives \\
by neurologist defined as:* & Ander legal protection \\
$-\quad$ Unilateral motor deficit with a score $\geq 5$ & expected to survive more than 90 days \\
& - Facial palsy (item 4 NIHSS 0 to 2 )
\end{tabular}

${ }^{*}$ New criteria of the version 3.0.

mRS, modified Rankin Scale; NIHSS, National Institutes of Health Stroke Scale.

possible. In the setting of no LVO, the patients are treated as per institutional standards.

\section{Clinical assessment}

Baseline characteristics include prestroke mRS score, symptoms and intrahospital time metrics. Neurological deficit is assessed using the NIHSS score at baseline, after $24( \pm 6)$ hours, at 5-7 days (or discharge if earlier) and at $90( \pm 15)$ days. At $90( \pm 15)$ days and $12( \pm 1)$ months, outcome assessment is also evaluated with the mRS score and health-related quality of life (EQ-5D-5L). ${ }^{1011}$

\section{Imaging protocol}

Baseline imaging (MRI or CT for the standard management group and CBCT and angiogram for the DAT group) is performed. Baseline imaging, angiographic imaging before, and at the end of endovascular procedure as well as follow-up imaging at $24( \pm 6)$ hours for ICH are assessed by an independent core laboratory. The core laboratory evaluates the findings on the baseline imaging for the ASPECTS (range 0-10, with 1 point subtracted for any evidence of early ischaemic change in each defined region on the CT scan or diffusion-weighted imaging sequence),${ }^{12}$ baseline vessel imaging (CT angiogram or MR angiogram) for the location of the occlusion. The core laboratory assessed also angiographic outcomes on digital subtraction angiography, using the modified Thrombolysis in Cerebral Infarction (mTICI) score, which ranges from 0 (no reperfusion) to 3 (complete reperfusion). ${ }^{13}$ Radiological outcome measures will be centrally analysed, blinded to treatment allocation.

\section{Outcomes}

\section{Primary outcome}

The proportion of patients with functional independence defined as mRS score 0-2 at $90 \quad( \pm 15)$ days between DAT and conventional admission in patients $\leq 75$ years old presenting an acute severe neurological deficit at prehospital stage and directly admitted at an endovascular-capable centre within 5 hours of onset.

\section{Secondary outcomes}

1. Secondary feasibility endpoints:

- Rate and site of LVO.

- Intrahospital time metrics (admission to imaging/ needle/puncture/reperfusion, imaging to puncture/reperfusion and puncture to reperfusion).

2. Secondary efficacy endpoints:

- Quality of reperfusion according to the mTICI score.

- Procedural complications (embolus in a new territory, perforation and dissection).

- Clinical status with the NIHSS score at $24( \pm 6)$ hours, 5-7 days (or discharge if earlier), $90( \pm 15)$ days.

- Blinded $12( \pm 1)$-month mRS score.

3. Secondary safety endpoints:

- Rate of patients eligible to IV rt-PA alone.

- Rate of ICH.

- Rate of stroke mimics.

- Rate of patients requiring secondary stroke imaging.

- Rate of intracerebral haemorrhagic transformation of ischaemic stroke according to the European Cooperative Acute Stroke Study III classification. ${ }^{14}$

- Rate of mortality at $90( \pm 15)$ days and $12( \pm 1)$ months.

- Rate of decompressive hemicraniectomy.

4. Cost-utility assessment include health-related quality of life assessment at $90( \pm 15)$ days and $12( \pm 1)$ months and assessment of costs from the time of randomisation to the 12-month follow-up:

- Costs of hospitalisation.

- Institutionalised living.

- Outpatient care. 
- Informal care provided by relatives.

- Cost of lost productivity.

\section{Recruitment}

The patients are expected to be included during a 30-month period starting in April 2020.

2016-2017: Protocol, approvals from ethics committee (CPP IDF I) and the French Medicine Agency (Agence Nationale de Sécurité du Médicament et des produits de santé, ANSM); trial tool development (online case report form (CRF) and randomisation system).

2020-2023: Inclusion of patients.

2023-2024: Cleaning and closure of the database, data analyses, writing of the manuscript and submission for publication.

\section{Trial status}

The current protocol is 2.0. Study started enrolment in 27 April 2020. To date (31 January 2021), seven patients have been randomised in the study (one centre open).

\section{Patient and public involvement}

Patients will not be invited to comment on study design or conduction of the trial.

\section{METHODS: ASSIGNMENT OF INTERVENTIONS Allocation and sequence generation}

After inclusion, the patients are randomised in two arms using a web-based centralised system with a 1:1 ratio to either DAT or standard management (figure 1). The randomisation sequence is provided by an independent statistician (who did not take part in assessing the patients at any point in the study) using computer-generated random numbers and stratified by centre and delay from onset to hospital admission (before or after 2.5 hours). The randomisation sequence is implemented in the electronic case report form system to ensure a centralised real-time randomisation procedure. Subjects are enrolled and randomised by emergency physicians, neurologists or neuroradiologists.

\section{Blinding}

For the primary outcome, a centralised certified clinical research nurse from the trial centre, who will be unaware of the treatment group assignments, records the mRS at 90 days and 1 year by telephone with the patient, proxy or healthcare provider. $^{89}$ All neuroimaging readings including determination of the ASPECTS score, arterial occlusion site, clot burden score and haemorrhagic transformation are performed by the imaging core laboratory, which is also blinded to procedure allocation.

\section{METHODS: DATA COLLECTION, MANAGEMENT AND ANALYSIS Data collection and management}

Similarly to other studies ${ }^{15}$ the entire study is conducted using eCRFs, where all clinical data on enrolled subjects are entered (single-keyed) by the site personnel. The
eCRF was developed using CleanWeb (Tentelemed) software. The essential data necessary for monitoring the primary and secondary endpoints are identified and managed at regular intervals throughout the trial. Data are monitored by members of the CIC 1433 Technological Innovation of Nancy University Hospital using the predefined rules and queries are automatically edited. Lastly, overall automated monitoring is performed by the data manager after completion of data entry. In cases of discrepancies, queries can be edited to resolve the problems encountered.

\section{Patient withdrawal}

Evaluated procedure is tested during the management of endovascular thrombectomy. Nevertheless, the participant can withdraw consent at any time without the need for further explanation. Data will be destroyed and a new patient will be randomised for the complete sample size.

\section{Statistical methods}

Sample size estimation

Based on the literature, ${ }^{25-7}$ we expect a 90-day mRS 0-2 rate of $30 \%$ in the control arm. We assume that DAT approach (intervention arm) will be associated with an absolute increase of $20 \%$ (corresponding to a 90-day mRS 0-2 rate of 50\%) due to 1-hour delay to reperfusion reduction. To detect this effect size, with a two-sided test at the 0.05 level of significance, and a power of $80 \%, 93$ subjects per arm will be required. To account for an anticipated rate of $10 \%$ dropout (ie, patients lost to follow-up and without LVO), we planned to include a total of 208 subjects (104 per arm).

\section{Interim analysis}

One interim analysis is planned once $50 \%$ of patients have been included, for the study to be stopped early owing either to compelling evidence of efficacy (using a prespecified Haybittle-Peto efficacy boundary with an alpha level of 0.001 ) or of futility. The independent data and safety monitoring board (DSMB) could recommend stopping the study if prolongation of the trial clearly compromises patient safety (in case of serious adverse reactions (SARs) or suspected unexpected serious adverse reactions (SUSARs)). The steering committee will be responsible to continue, hold or stop the study based on the DSMB recommendations.

\section{Statistical analyses}

Statistical analyses will be carried out independently by the CIC 1433 Technological Innovation of Nancy University hospital under the responsibility of Professor Jacques Felblinger, where statisticians and investigators will be aware of the treatment group allocation. Baseline characteristics will be described for each treatment group; categorical variables will be expressed as frequencies and percentages and quantitative variables will be expressed as means $\pm \mathrm{SD}$ or medians (IQR) for non-Gaussian distribution. Normality of distributions will be assessed graphically and by using the Shapiro-Wilk test. No formal 
statistical comparisons of baseline characteristics will be done; clinical importance of any imbalance will be noted. All analyses will be performed using all randomised participants based on their original group of randomisation, according to the intention-to-treat principle. The intention-to-treat analysis will analyse all included patients and patients will be analysed according to the randomisation scheme. This analysis will include all patients with LVO but also with ICH, stroke mimics, ischaemic stroke without LVO at admission, independently of receiving MT or not. A per-protocol analysis will be considered only for primary endpoint as a secondary analysis. Perprotocol population will include all randomised patients excluding those without LVO strokes. Furthermore, the costs avoided analysis will take into account a cost difference between the two randomisation arms at 12 $( \pm 1)$ months and will extrapolate this difference over an expected lifetime using a Markov model. The cost-utility analysis will also be performed with health-related quality of life estimated with EQ-5D-5L questionnaire. The CONSORT statement recommendations will be applied for drafting the final report.

\section{METHODS: MONITORING \\ Data monitoring}

Before the start of the study, neurological and neuroradiological medical and paramedical teams are trained at each site for the study protocol by study coordinators. Physicians are in charge of patient screening and inclusion. Data will be collected in a web-based eCRF by trial personnel. Each centre will only have access to sitespecific data. Each patient will receive a unique trial identification number. Only the investigators and research team will have access to any protected health information of study participants and any study data.

Data monitoring and quality control will be conducted in each centre after the first 10 inclusions then after the next 20 inclusions and at the end of the study by official representatives of the study promoter (Department of Clinical Research and Innovation, Nancy University Hospital). Data will be handled according to the French law. All original records (including consent forms, reports of SUSARs and relevant correspondences) will be archived at trial sites for 15 years. The clean trial database file will be anonymised and maintained for 15 years. Only the principal investigators and the statistician will have access to the final dataset.

\section{Harms}

Every adverse event that could be related to the trial will be reported to the trial coordinating centre. According to the French law, all suspected serious adverse events will be reported to the ANSM. The DSMB will also be informed. DSMB is independent from the trial investigators and will perform an ongoing review of safety parameters and study conduct. The members of the DSMB are not participants of the DIRECT ANGIO consortium and not involved in the clinical trial. The DSMB is composed of one neuroradiologist, one pharmacovigilance specialist and one methodologist, who are not participating in the study and are not affiliated with the sponsor and who have skills and expertise in clinical neuroscience and clinical research. The DSMB will be responsible for safeguarding the interests of trial participants, assessing the safety of the interventions during the trial and for monitoring the overall conduct of the trial. DSMB could also formulate recommendations related to the recruitment/retention of participants, their management, improving adherence to protocol-specified regimens, and the procedures for data management and quality control. No formal criteria are set to stop the study. However, recommendations for pausing or stopping the study could be made by DSMB in case of SARs and SUSAR. The scientific committee will be responsible for promptly reviewing the DSMB recommendations and to decide whether to continue, hold or stop the study, and to determine whether amendments to the protocol are needed.

\section{ETHICS AND DISSEMINATION}

Any change to eligibility criteria, outcomes and analyses will be communicated to investigators, the ethics committee and the ANSM to obtain their approval. The DIRECT ANGIO trial was approved by the ethics committee (CPP) of Ile de France 1.

\section{Consent or assent}

Whenever possible to include the patient, written inform consent will be searched. Nevertheless, related to neurological injury and emergency, the patient may be unable to provide written informed consent. In this case, written informed consent could be obtained from the patient next of kin if immediately available. Otherwise, an emergency consent procedure is used with investigator signature countersigned by an independent physician. As soon as possible after recovery, written informed consent form will be obtained.

\section{Dissemination}

Results will be published in an international peer-reviewed medical journal.

\section{DISCUSSION}

Few studies evaluated the impact of DAT in the management of suspected LVO stroke patients, especially in the setting of primary admission. DIRECT ANGIO is a French PROBE, two-arm randomised trial comparing DAT versus standard management for patients with acute severe neurological deficit before hospital admission and thus suspected to anterior circulation LVO eligible to MT. Randomisation is performed before hospital admission and within 5 hours of onset. Primary endpoint is the 90-day functional independence. The study will provide efficacy and safety data as well as socioeconomic evidence 
for the DAT management for patients with acute severe stroke.

\section{Author affiliations}

${ }^{1}$ Neurology, Stroke Unit, CHRU de Nancy, Nancy, Lorraine, France

${ }^{2}$ Neuroradiology, CHRU de Nancy, Nancy, Lorraine, France

${ }^{3}$ INSERM U1254, CHU Nancy, Vandoeuvre-lès-Nancy, France

${ }^{4}$ INSERM CIC-IT 1433, CHU Nancy, Vandœuvre-lès-Nancy, France

${ }^{5}$ Urgences, CHRU de Nancy, Nancy, Lorraine, France

${ }^{6}$ Hôpital Central, Service d'Anesthésie-Réanimation, CHRU Nancy, Nancy, France

${ }^{7}$ CIC-1433 Epidémiologie clinique, Centre Hospitalier Universitaire de Nancy,

Vandoeuvre-lès-Nancy, France

${ }^{8}$ Neuroradiology, Centre Hospitalier Universitaire de Montpellier, Montpellier, France

${ }^{9}$ Neurology, Stroke Unit, CHU Montpellier, Montpellier, Languedoc-Roussillon, France

${ }^{10}$ Urgences, CHU Montpellier, Montpellier, Languedoc-Roussillon, France

${ }^{11}$ Neuroradiology, Azienda Ospedaliero Universitaria Careggi, Firenze, Italy

${ }^{12}$ Neuroradiology, Hopital Foch, Suresnes, France

${ }^{13}$ Neurology, Stroke Unit, Hôpital Foch, Suresnes, Île-de-France, France

${ }^{14}$ SAMU 92, APHP, Paris, île-de-France, France

${ }^{15}$ Interventional Neuroradiology, CHU Limoges, Limoges, Limousin, France

${ }^{16}$ Neurology, Stroke Unit, CHU Limoges, Limoges, Limousin, France

${ }^{17}$ SAMU, CHU Limoges, Limoges, Limousin, France

${ }^{18}$ Neuroradiology, CHU Besancon, Besancon, France

${ }^{19}$ Neurology, Stroke Unit, CHU Besancon, Besancon, France

${ }^{20}$ Emergency, University of Franche Comté, Besançon, France

${ }^{21}$ Neuroradiology, CHU de Bordeaux, Bordeaux, Aquitaine, France

${ }^{22}$ Neurology, Stroke Unit, CHU Bordeaux, Bordeaux, France

${ }^{23}$ Anesthesiology, CHU de Bordeaux, Bordeaux, Aquitaine, France

${ }^{24}$ Neuroradiology, CH Colmar, Colmar, Alsace, France

${ }^{25}$ Neurology, Stroke Unit, CH Colmar, Colmar, Alsace, France

${ }^{26}$ SAMU, CH Colmar, Colmar, Alsace, France

${ }^{27}$ Neuroradiology, CHU Reims, Reims, Champagne-Ardenne, France

${ }^{28}$ Neurology, Stroke Unit, CHU Reims, Reims, Champagne-Ardenne, France

${ }^{29}$ Urgences, CHU Reims, Reims, Champagne-Ardenne, France

${ }^{30}$ Interventional Neuroradiology, Fondation Rothschild, Paris, Île-de-France, France

${ }^{31}$ Neurology, Stroke Unit, Fondation Rothschild, Paris, Île-de-France, France

${ }^{32}$ Neurology, Stroke Unit, GHR Mulhouse Sud Alsace, Mulhouse, France

${ }^{33} \mathrm{CIC}$ EC, CHRU de Nancy, Nancy, Lorraine, France

Twitter Thibaut Desmettre @TDesmettre

Acknowledgements DIRECT ANGIO boards and institutions: DSMB, CHRU Nancy, CIC-IT, CIC-EC, DRI.

Collaborators DIRECT ANGI0 Investigators: CHRU-Nancy: Benjamin Gory, Isabelle Costa, Serge Bracard, René Anxionnat, Marc Braun, Anne-Laure Derelle, Romain Tonnelet, Liang Liao, François Zhu, Emmanuelle Schmitt, Sophie Planel, Sébastien Richard, Lisa Humbertjean, Gioia Mione, Jean-Christophe Lacour, Nolwenn RiouComte, Gabriela Hossu, Marine Beaumont, Mitchelle Bailang, Gérard Audibert, Marie Reitter, Agnès Masson, Lionel Alb, Adriana Tabarna, Marcela Voicu, Iona Podar, Madalina Brezeanu. CHU Besançon: Alessandra Biondi, Giovanni Vitale, Fortunato Di Caterino, Guillaume Charbonnier, Panagiotis Primikiris, Thierry Moulin, Elisabeth de Bustos Medeiros, Benjamin Bouamra, Louise Bonnet, Fabrice Vuillier, Thibaut Desmettre CH Colmar: Pablo A Lebedinsky, Mariano Musacchio, Frederico Bolognini, Francis Vuillemet, Nicolas Kempf, CH Mulhouse: Elie Cohen-Khallas, Andreas Fickl, Eric Schluck, Mathilde Goudot, Sylvie Courtois, Sohrab Mostoufizadehghalamfarsa, Alessandro La Porta. CHU Reims: Sébastien Soize, Solène Moulin, Laurent Pierot, Pierre-François Manceau, Maher Sahnoun, Christophe Gelmini, Nathalie Caucheteux, Vi Tuan Hua, Isabelle Serre, Mickaël Hoang. CHU Bordeaux: Catherine Pradeau, Gaultier Marnat, Florent Gariel, Xavier Barreau, Jérôme Berge, Louis Veunac, Patrice Menegon, Igor Sibon, Ludovic Lucas, Stéphane Olindo, Pauline Renou, Sharmila Sagnier, Mathilde Poli, Sabrina Debruxelles, Jean-Marc Mene, Musa Sesay. CHU Montpellier: Vincent Costalat, Caroline Arquizan, Cyril Dargazanli, Grégory Gascou, Pierre-Henri Lefèvre, Imad Derraz, Carlos Riquelme, Nicolas Gaillard, Isabelle Mourand, Lucas Corti. Fondation Adolphe de Rothschild: Michel Piotin, Raphael Blanc, Hocine Redjem, Simon Escalard, Jean-Philippe Desilles, Gabriele Ciccio, Stanislas Smajda, Mikael Mazighi, Mikael Obadia, Candice Sabben, Ovide Corabianu, Thomas de Broucker, Didier Smadja, Sonia Alamowitch, Olivier Ille, Eric Manchon, Pierre-Yves Garcia, Guillaume Taylor, Malek Ben Maacha. Hôpital Foch: Adrien Wang, Serge Evrard, Maya Tchikviladze, Nadia Ajili, Bertrand Lapergue, David Weisenburger, Lucas Gorza, Oguzhan Coskun, Arturo Consoli, Federico
Di Maria, Georges Rodesh, Morgan Leguen, Julie Gratieux, Fernando Pico, Haja Rakotoharinandrasana, Philippe Tassan, Roxanna Poll, Sylvie Marinier. CHU Limoges: Aymerci Rouchaud, Suzana Saleme, Charbel Mounayer, Francisco Macian-Montoro, Dominique Cailloce.

Contributors NR-C, ACh, SR, LN, FG, GH and BG are members of DIRECT ANGIO scientific committee and contributed to the conception and design of the research protocol. NR-C, ACh, SR and BG provided critical skills concerning trial interventions and procedures. NR-C and BG wrote the first version of the protocol. $B G$ wrote this manuscript. GH designed the statistical analysis plan. NR-C, FZ, ACh, SR, FG, GH and $B G$ are involved in acquisition, analysis and interpretation of the data. NR-C, FZ, SR, LN, GA, HA, VC, CA, OB, ACo, BL, TL, AR, FM, DC, AB, TM, TD, GM, IS, XC, APL, $\mathrm{FV}, \mathrm{NK}, \mathrm{LP}, \mathrm{SM}, \mathrm{PL}, \mathrm{MM}, \mathrm{RB}, \mathrm{CS}, \mathrm{ES}, \mathrm{SB}, \mathrm{RA}$ and $\mathrm{BG}$ are involved in acquisition of the data. All authors revised the final protocol and approved this submission.

Funding DIRECT ANGI0 trial was supported by funding from French Ministry of Health (Programme Hospitalier de Recherche Clinique Inter Régional (PHRC IR 2018) and from the university hospital of Nancy (principal investigator: BG. Funding amount: 295990 euros).

Disclaimer The funder had no role in study design, study conduction, writing or submitting the manuscript.

Competing interests None declared.

Patient and public involvement Patients and/or the public were involved in the design, or conduct, or reporting or dissemination plans of this research. Refer to the Methods and analysis section for further details.

Patient consent for publication Not required.

Provenance and peer review Not commissioned; externally peer reviewed

Open access This is an open access article distributed in accordance with the Creative Commons Attribution Non Commercial (CC BY-NC 4.0) license, which permits others to distribute, remix, adapt, build upon this work non-commercially, and license their derivative works on different terms, provided the original work is properly cited, appropriate credit is given, any changes made indicated, and the use is non-commercial. See: http://creativecommons.org/licenses/by-nc/4.0/.

\section{ORCID IDs}

Sébastien Richard http://orcid.org/0000-0002-0945-5656

Benjamin Gory http://orcid.org/0000-0001-8424-4464

\section{REFERENCES}

1 Waqas M, Rai AT, Vakharia K, et al. Effect of definition and methods on estimates of prevalence of large vessel occlusion in acute ischemic stroke: a systematic review and meta-analysis. $J$ Neurointerv Surg 2020;12:260-5.

2 Bracard S, Ducrocq X, Mas JL, et al. Mechanical thrombectomy after intravenous alteplase versus alteplase alone after stroke (THRACE): a randomised controlled trial. Lancet Neurol 2016;15:1138-47.

3 Saver JL, Goyal M, van der Lugt A, et al. Time to treatment with endovascular thrombectomy and outcomes from ischemic stroke: a meta-analysis. JAMA 2016;316:1279-88.

4 Campbell BCV, Majoie CBLM, Albers GW, et al. Penumbral imaging and functional outcome in patients with anterior circulation ischaemic stroke treated with endovascular thrombectomy versus medical therapy: a meta-analysis of individual patient-level data. Lancet Neurol 2019;18:46-55.

5 Psychogios M-N, Behme D, Schregel K, et al. One-stop management of acute stroke patients: minimizing door-to-reperfusion times. Stroke 2017;48:3152-5.

6 Ribo M, Boned S, Rubiera M, et al. Direct transfer to angiosuite to reduce door-to-puncture time in thrombectomy for acute stroke. $J$ Neurointerv Surg 2018;10:221-4.

7 Jadhav AP, Kenmuir CL, Aghaebrahim A, et al. Interfacility transfer directly to the neuroangiography suite in acute ischemic stroke patients undergoing thrombectomy Stroke 2017:48:1884-9.

8 Schulz KF, Altman DG, Moher D, et al. Consort 2010 statement: updated guidelines for reporting parallel group randomised trials. BMJ 2010;340:c332.

9 Chan A-W, Tetzlaff JM, Gøtzsche PC, et al. Spirit 2013 explanation and elaboration: guidance for protocols of clinical trials. BMJ 2013;346:e7586

10 van Swieten JC, Koudstaal PJ, Visser MC, et al. Interobserver agreement for the assessment of handicap in stroke patients. Stroke 1988;19:604-7. 
11 EuroQol Group. EuroQol--a new facility for the measurement of health-related quality of life. Health Policy 1990;16:199-208.

12 Barber PA, Demchuk AM, Zhang J, et al. Validity and reliability of a quantitative computed tomography score in predicting outcome of hyperacute stroke before thrombolytic therapy. aspects Study Group. Alberta stroke programme early CT score. Lancet 2000;355:1670-4.

13 Zaidat OO, Yoo AJ, Khatri P, et al. Recommendations on angiographic revascularization grading standards for acute ischemic stroke: a consensus statement. Stroke 2013;44:2650-63.
14 Hacke W, Kaste M, Bluhmki E, et al. Thrombolysis with alteplase 3 to 4.5 hours after acute ischemic stroke. N Engl J Med 2008;359:1317-29.

15 Chabanne R, Fernandez-Canal C, Degos V, et al. Sedation versus general anaesthesia in endovascular therapy for anterior circulation acute ischaemic stroke: the multicentre randomised controlled AMETIS trial study protocol. BMJ Open 2019;9:e027561. 